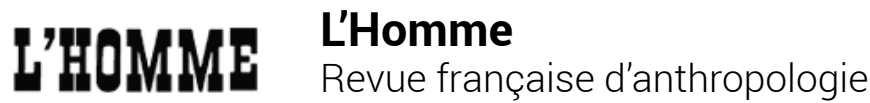

231-232| 2019

Cumulus: Hoarding, Hosting, Hospitality

\section{Olivier Keller, L'Invention du nombre. Des mythes de création aux Éléments d'Euclide}

Danièle Dehouve

\section{(2) OpenEdition \\ 1 Journals}

\section{Édition électronique}

URL : https://journals.openedition.org/lhomme/35754

DOI : 10.4000//homme.35754

ISSN : 1953-8103

Éditeur

Éditions de l'EHESS

\section{Édition imprimée}

Date de publication : 21 novembre 2019

Pagination : 302-305

ISBN : 978-2-7132-2794-3

ISSN : 0439-4216

\section{Référence électronique}

Danièle Dehouve, "Olivier Keller, L'Invention du nombre. Des mythes de création aux Éléments d'Euclide », L'Homme [En ligne], 231-232 | 2019, mis en ligne le 21 novembre 2019, consulté le 23 avril 2022. URL : http://journals.openedition.org/lhomme/35754; DOI : https://doi.org/10.4000/lhomme.35754

Ce document a été généré automatiquement le 23 avril 2022.

(C) École des hautes études en sciences sociales 


\title{
Olivier Keller, L'Invention du nombre. Des mythes de création aux Éléments d'Euclide
}

\author{
Danièle Dehouve
}

\section{RÉFÉRENCE}

Olivier Keller, L'Invention du nombre. Des mythes de création aux Éléments d'Euclide. Paris, Classiques Garnier, 2016, 343 p., bibl., index, ill. (« Histoire et philosophie des sciences » 12).

1 Ce livre d'Olivier Keller n'est pas le premier à retracer les grandes étapes de l'invention des nombres, mais les précédents ouvrages se présentaient comme des histoires du savoir-compter, en quête des prémices des outils mathématiques dont nous disposons de nos jours ${ }^{1}$. L'approche de Keller est tout autre. S'appuyant sur ses travaux antérieurs concernant l'histoire de la géométrie ${ }^{2}$, ce mathématicien de formation assure que «l'origine de l'arithmétique n'est pas plus dans le commerce et la comptabilité que l'origine de la géométrie n'est dans la mesure des terrains » (p.7). Il nous offre un essai de philosophie des sciences qui revisite des thèmes classiques de l'anthropologie mythes de création, "classifications primitives ", rituels, divination et numérologie -, à partir d'un questionnement issu de la contradiction fondamentale « un-multiple ». En effet, lorsque nous disons " un et un font deux ", un et un sont " le même absolument " et " différents absolument »: « Le nombre ne peut exister sans le "un" qui est en même temps "des uns", c'est-à-dire sans le un qui est en même temps multiple » (p. 12). Pour Olivier Keller, l'histoire de l'invention du nombre est celle du déploiement de la réflexion humaine sur la contradiction fondamentale de l'un-multiple.

2 En premier lieu, le chercheur aborde sous cet angle la question des «classifications primitives ", objet anthropologique s'il en est. La pensée archaïque, dit-il, saisit le monde comme un tout (c'est l'Un), mais aussi dans sa diversité (c'est le Multiple), ce qui 
donne lieu à plusieurs formes phénoménales. Olivier Keller commence par citer l'« animisme vague », qui résulte de la conscience d'une essence unique de la variété du monde tout en mettant l'accent sur le Tout : «l'un multiple est bien là, mais le multiple ne se manifeste encore que comme l'apparence de l'un ; au fond, il n'y a guère que le Tout » (p. 41). Il poursuit avec l'« animisme pluriel », qui pense la multiplicité et recrée l'unité des choses en les reliant à partir d'une débauche d'analogies arbitraires : cette fois-ci, c'est le multiple qui prévaut, tout est encore informel et éparpillé. Avec le "totémisme ", l'animisme pluriel s'ordonne au moyen de correspondances spécifiques entre le monde humain et naturel, mais le multiple domine encore. La synthèse de la totalité n'est atteinte qu'à l'aide des "classifications totalitaires » qui organisent ces correspondances de façon systématique sous deux formes : les classifications en arbres ou en tableaux (par exemple, celle par points cardinaux). Ainsi Olivier Keller fait-il la preuve que la contradiction un-multiple est une des manières d'aborder le sujet des « classifications primitives» sur lequel se sont illustrés de grands noms ${ }^{3}$.

On trouve encore l'un-multiple dans les mythes de genèse. Ce qui existe était à l'origine un agglomérat qu'il a fallu dissocier. Pour cela, plusieurs procédés ont été utilisés, qui peuvent coexister au sein d'une même population : la séparation du ciel et de la terre est un schéma universel, de même que le corps démantelé et la déambulation créatrice. Le fameux démiurge des Iqwaye de Nouvelle-Guinée popularisé par Jadran Mimica ${ }^{4} \mathrm{y}$ occupe une place de choix: Omalyce était représenté par un corps clos sur lui-même, son pénis ou cordon ombilical dans sa bouche; lorsque Omalyce a coupé le cordon ombilical, le ciel et la terre se sont séparés, ses yeux sont devenus étoiles, soleil et lune et, en vomissant, il a créé toutes les choses. Cela explique que les dieux soient partout à la fois singuliers et pluriels. Enfin, de la création, on aboutit aux cycles de création : les cycles représentent l'expression temporelle de l'un-multiple, l'année qui revient est à la fois autre et identique ; et d'un cycle on passe à une succession de cycles emboîtés qui prennent place dans un cycle supérieur.

4 «Sur des modes divers, la genèse archaïque est donc l'un qui actualise sa multiplicité intrinsèque. Mais comment sortir du quantitatif de l'auto-démultiplication?» (p. 125). Comment exprimer la diversification? Par ces formes hybrides que Keller nomme les " quanta » pour éviter de les appeler des «nombres », si bien qu'il propose " dyade » au lieu de 2, «triade » au lieu de 3, «tétrade » au lieu de 4, «pentade » au lieu de 5, etc. Un «quantum » est l'expression quantitative d'une qualité, qui renvoie le plus souvent aux genèses. Ainsi, dans beaucoup de populations amérindiennes, la tétrade est omniprésente. Chez les Sioux Oglala, par exemple, la différenciation primordiale est celle de Vent en quatre vents correspondant aux quatre directions; en conséquence de quoi, les hommes découpent tout (dieux ou périodes de la vie humaine) en paquets de quatre. Il ne s'agit pas d'un procédé par analogie ( on ne voit pas comment attribuer raisonnablement une direction géographique à la lunaison, à l'année, aux fruits, aux animaux rampants, à chaque pouce, etc.», p. 129), mais de l'expression d'une qualité : la perfection de la création. Cependant, le quantum n'a pas seulement une existence sacrée liée aux genèses; il peut aussi s'exprimer dans des domaines plus profanes, lorsque le nombre désigne n'importe quelle qualité - individus, choses ou périodes de temps.

Olivier Keller propose ici de considérer le quantum et le nombre comme deux catégories analytiques distinctes. Les quanta, puisqu'il n'y a entre eux aucun lien de caractère numérique, sont indifférents les uns par rapport aux autres ; par exemple, un 
nombre plus élevé ne communique pas une plus grande valeur qu'un nombre plus petit. Qui plus est, pour exister, les quanta n'ont pas besoin de système numérique, il suffit de pratiquer une «bijection ", c'est-à-dire une correspondance un à un entre des objets et une collection type ; "pour compter je dois certainement faire une bijection, mais je n'ai pas besoin de savoir compter pour mettre un verre devant chaque assiette de la table» (p. 18). De cette façon, la tétrade des Amérindiens suppose une bijection entre les quatre directions du monde et les paquets de quatre éléments qui servent à découper les choses les plus diverses. Le nombre, quant à lui, est un «système autonome et déqualifié de quanta » (p. 137). «Privées de toute référence autre qu'ellesmêmes, les multiplicités doivent se déployer les unes par rapport aux autres » (p. 175). Cela se produit lorsqu'une multiplicité représente un «un» d'un ordre supérieur. À mon avis, la démonstration de Keller aurait été simplifiée par l'emploi du terme "base », que l'auteur a évité, j'ignore pour quelle raison. On sait, en effet, que tous les systèmes numériques utilisent des bases diverses. Dans un système à base 10 comme le nôtre, 10 constitue une unité d'ordre supérieur, de même que 100 et 1000: en conséquence, un est "un », et 10, 100 et 1000 constituent chacun un autre " un ». Ce système auto-référent fonctionne sur la base du calcul.

6 L'auteur envisage ensuite l'invention du nombre rationnel, que l'on peut étudier en Égypte et en Mésopotamie en raison de la documentation que nous ont laissée ces empires. Un nombre rationnel est un nombre qui peut être écrit en une fraction de deux nombres rationnels. Cette invention démontre concrètement l'identité des différents nombres : par là, «le nombre réalise complètement l'un-multiple qui lui a donné naissance » (p. 201). C'est à Euclide qu'il reviendra d'en faire la théorie dans le Livre VII des Éléments. Le dernier chapitre traite de la numérologie qui se développe à partir des systèmes numériques et de la conception pythagoricienne selon laquelle «toutes choses sont nombres ». On la retrouve dans diverses pratiques divinatoires, la période védique de l'Inde ou encore le pythagorisme.

7 L'argument développé par l'auteur tout au long de l'ouvrage va de pair avec une opinion tranchée sur l'existence du «sens du nombre», cette fameuse prédisposition innée à la numération dont Stanislas Dehaene s'est fait le chantre ${ }^{5}$. Des expériences pratiquées sur de très jeunes enfants, ainsi que sur certains animaux, montreraient que cette prédisposition se situe dans le cerveau. Olivier Keller s'inscrit en faux contre cette école puisque, selon lui, le nombre est une invention qui résulte du travail social mené durant des générations sur la contradiction entre l'un et le multiple. Signalons que, dans le débat international qu'animent aujourd'hui les cognitivistes, la posture de Keller est confortée par des prises de position de poids, telle que celle de Rafael Nuñez pour qui il est nécessaire de différencier le sens de la quantité du sens numérique, seul le premier étant inné6.

8 Enfin, on ne saurait clore cette recension sans aborder de front le «sujet qui peut fâcher " des anthropologues. Olivier Keller présente une histoire du nombre, c'est-àdire un cheminement évolutif. Or, la pratique concrète de l'ethnologue le conduit à voir les choses dans la synchronie. Par exemple, si je relis mon ouvrage sur le nombre chez les Aztèques au $\mathrm{XVI}^{\mathrm{e}}$ siècle $^{7}$ en utilisant les métamorphoses de l'un-multiple proposées par Keller, il s'avère que celles-ci sont toutes présentes dans la même société au même moment. Les Aztèques possédaient, en effet, des mythes d'origine exprimant le passage de l'un au multiple au travers de récits de séparation du ciel et de la terre, et de corps démembrés. Ils disposaient d'un système élaboré de quanta et, surtout, la tétrade que 
j'ai appelée « cosmogramme ». S'ajoutait à cela un système numérique à bases 20, 400 et 8000 , et une numérologie qui s'exerçait dans la divination, en particulier liée aux cycles de 260 jours. Il faut préciser qu'un système complexe de rituels à quanta perdure aujourd'hui dans les communautés indiennes mexicaines ${ }^{8}$. Les travaux du mathématicien m'offrent donc des catégories analytiques permettant de mettre de l'ordre dans le foisonnement des usages numériques observés et de les envisager d'emblée d'un point de vue comparatiste. Mais je les utilise de manière synchronique, alors qu'elles ont été déduites par le chercheur d'un raisonnement logique se situant dans la chronologie. Cette situation me fait penser à la façon dont l'anthropologie peut mettre à profit le modèle diachronique élaboré par Arthur Hocart ${ }^{9}$. Ce Britannique spécialiste des îles Fidji et de Ceylan (aujourd'hui Sri Lanka) est l'auteur d'une théorie de la formation de l'État à partir de différenciations successives opérées sur la personne du roi sacré. Celui-ci est en effet doté d'une série de fonctions : personnage central du rituel, il imite le cours du soleil, il est garant de la fécondité, juge, guerrier, prêtre... Or, ces multiples fonctions peuvent, par dédoublements successifs, se distribuer entre des individus différents. Si Hocart situait son raisonnement dans un cadre évolutif, j'ai montré que son modèle était tout à fait adéquat pour expliquer le fonctionnement de sociétés à royauté sacrée en des termes qui n'ont en rien besoin d'une chronologie ${ }^{10}$. C'est la même fécondité que je trouve dans les propositions de Keller, qui se présentent, certes, comme un développement logique placé dans un cadre chronologique, mais se prêtent aussi à un usage synchronique et comparatiste. Et j'ajouterai que le processus de différenciation de la royauté sacrée selon Hocart peut être enrichi par la perspective de Keller du passage de l'un au multiple, puisque c'est effectivement de cela qu'il s'agit dans les dédoublements successifs des personnages royaux.

Enfin, la conclusion d'olivier Keller représente la critique la plus radicale que j'ai eu l'occasion de lire à l'encontre d'un leitmotiv qui, depuis Lévy-Bruhl, parcourt sous des formes diverses la littérature anthropologique: notre société fonctionnerait selon le principe de non-contradiction, au contraire des peuples que nous étudions. Pour Olivier Keller, à l'inverse, cette position relève de "l'aveuglement contemporain dans sa croyance à l'éradication de la contradiction dans "notre pensée" » (p. 282). «Plutôt que de s'accrocher au "principe de non-contradiction", il faudra reconnaître que l'unité des contraires est un principe. L'histoire, ouvertement, et l'axiomatique, à son corps défendant, s'accordent sur ce point » (p. 283). Dès les premiers mythes, la possibilité de concevoir le monde réside dans la faculté humaine à forger le concept contradictoire de l'un-multiple, capacité qui, loin de disparaître, a permis les développements mathématiques contemporains.

\section{NOTES}

1. Cf. : Karl Menninger, Number Words and Number Symbols. A Cultural History of Numbers, Cambridge, Mit Press, 1969 [1934] ; Geneviève Guitel, Histoire comparée des numérations écrites, Paris, Flammarion, 1975 (« Nouvelle bibliothèque scientifique »); Georges Ifrah, 
Histoire universelle des chiffres. L'Intelligence des hommes racontée par les nombres et le calcul, Paris, Robert Laffont, 1994 [1981], 2 vol. («Bouquins ») et Les Chiffres ou l'Histoire d'une grande invention, Paris, Robert Laffont, 1985 (« La Fontaine des sciences »).

2. Cf. : Olivier Keller, Aux origines de la géométrie. Le Paléolithique et le monde des chasseurscueilleurs, préf. de Denis Vialou, Paris, Vuibert, 2004 («Préhistoire des sciences »); et Une archéologie de la géométrie. La figure et le monde: peuples paysans sans écriture et premières civilisations, préf. d'Évelyne Barbin, Paris, Vuibert, 2006.

3. Olivier Keller cite, entre autres, les œuvres classiques d'Émile Durkheim et Marcel Mauss («De quelques formes primitives de classification [1903]», in Marcel Mauss, Euvres, éd. par Victor Karady, Paris, Minuit, 1969 : II, 13-89), et de Claude Lévi-Strauss (La Pensée sauvage, Paris, Plon, 1962). Personnellement, j'ajouterai que des traits relevés par Philippe Descola (notamment dans Philippe Descola, ed., La Fabrique des images. Visions du monde et formes de la représentation [exposition, Paris, musée du quai Branly, février 2010-juillet 2011], Paris, Somogy-Musée du quai Branly, 2010) comme caractéristiques de ses quatre ontologies figurent dans le modèle de Keller, agencés et pensés autrement.

4. Cf. Jadran Mimica, Intimations of Infinity. The Mythopoeia of the Iqwaye Counting System and Number, Oxford-New York, Berg, 1988 ("Explorations in Anthropology»). Rappelons que Mimica voit dans ce mythe l'image de l'invention du système numérique.

5. Cf. Stanislas Dehaene, La Bosse des maths. Quinze ans après, Paris, Odile Jacob, 2010.

6. Cf. Rafael E. Nuñez, «Is There Really an Evolved Capacity for Number?", Trends in Cognitive Sciences, 2017, 21 (6) : 409-424.

7. Danièle Dehouve, L'Imaginaire des nombres chez les anciens Mexicains, Rennes, Presses universitaires de Rennes, 2011 ("Sciences des religions »). J'utilise ici la terminologie de Keller, mais je ne la connaissais pas lorsque j'ai rédigé l'ouvrage.

8. Cf. Danièle Dehouve, Des nombres pour les dieux, Orsay, Tonaltepec prod., 2010, film de $48 \mathrm{mn}$. En ligne : https://www.nakala.fr/nakala/data/11280/91407a68

9. Cf. Arthur M. Hocart, Rois et courtisans, Paris, Le Seuil, 1978 («Recherches anthropologiques ») [trad. de Kings and Councillors, Le Caire, Printing Office Paul Babey, 1936].

10. Cf. Danièle Dehouve, Essai sur la royauté sacrée en République mexicaine, Paris, CNRS

Éd., 2006 (« Anthropologie ») : 25-32. 